\title{
High expression of MAGE-A9 is associated with unfavorable survival in esophageal squamous cell carcinoma
}

\author{
YU QI $^{1}, \mathrm{KE} \mathrm{XIN} \mathrm{CAO}^{1}$, FU CHEN XING ${ }^{1}, \mathrm{CHUN} \mathrm{YANG} \mathrm{ZHANG}^{1}$, QI HUANG $^{1}$, \\ KAI WU ${ }^{1}$, FENG BIAO WEN ${ }^{1}$, SONG ZHAO ${ }^{1}$ and $\mathrm{XIN} \mathrm{LI}^{2}$ \\ Departments of ${ }^{1}$ Thoracic Surgery and ${ }^{2}$ Oncology, The First Affiliated Hospital, \\ Zhengzhou University, Zhengzhou, Henan 450000, P.R. China
}

Received November 23, 2015; Accepted April 24, 2017

DOI: $10.3892 / \mathrm{ol} .2017 .6614$

\begin{abstract}
Melanoma-associated antigens (MAGEs) are a group of well-characterized members of the cancer/testis antigen family, which are expressed in a variety of malignant tumors. MAGE-A9, a subfamily of MAGE-As, has been studied in a number of types of cancer and have been associated with unfavorable survival outcome. However, the expression of MAGE-A9 in human esophageal squamous cell carcinoma (ESCC) and association of MAGE-A9 with the clinicopathological characteristics of ESCC, particularly prognostic characteristics, remains unknown. The present study aimed at determining the expression level of MAGE-A9 and at evaluating its clinical significance in human ESCC. Quantitative polymerase chain reaction (qPCR) and immunohistochemistry (IHC) analyses were performed to characterize the expression of MAGE-A9 in ESCC tissues. Kaplan-Meier estimator survival and Cox's regression analyses were used to evaluate the prognosis of 103 patients with ESCC. The results of qPCR and IHC analysis revealed that the expression of MAGE-A9 was significantly increased in ESCC tissues, compared with that in healthy tissues. Furthermore, the expression level of MAGE-A9 protein in ESCC was significantly associated with the pathological grade $(\mathrm{P}=0.008)$, tumor size $(\mathrm{P}=0.027)$ and lymph node metastasis $(\mathrm{P}=0.009)$. Multivariate analysis using Cox's regression model identified that the expression level of MAGE-A9 and lymph node metastasis were independent prognostic factors for the overall survival rate of patients with ESCC $(\mathrm{P}=0.006$ and $\mathrm{P}=0.001$, respectively). The results of the present study are, to the best of our knowledge, the first to indicate that MAGE-A9 expression is a valuable prognostic biomarker for ESCC and that it may serve as a targeted therapy in the treatment of ESCC.
\end{abstract}

Correspondence to: Professor Xin Li, Department of Oncology, The First Affiliated Hospital, Zhengzhou University, 1 Jianshe Road, Zhengzhou, Henan 450000, P.R. China

E-mail: 578348982@qq.com

Key words: esophageal squamous cell carcinoma, melanomaassociated antigen A9
Increased expression of MAGE-A9 indicated an unfavorable survival outcome in patients with ESCC.

\section{Introduction}

Cancer is currently the second leading cause of human mortalities worldwide and is expected to surpass heart diseases as the leading cause of human mortality in the next few years. Esophageal cancer is the seventh leading cause of human cancer-associated mortality worldwide (1). Esophageal squamous cell carcinoma (ESCC) is the most major histological type of esophageal cancer. In China, the annual incidence and mortality rates of esophageal carcinoma rank fifth and fourth, respectively (2). The majority of patients with ESCC are diagnosed at an advanced stage of the disease, despite the development of rapid advances in surgical techniques, radiotherapy and chemotherapy, and the 5-year survival rate remains poor (between 10 and 30\%) (3). ESCC is hypothesized to develop due to risk factors including the accumulation of genetic mutations, tobacco, alcohol consumption, consuming hot food or water frequently, a low intake of fresh fruit and vegetables, obesity and poor diet, local environmental conditions and lifestyle (4). There are a limited number of specific biomarkers available for diagnostic use and targeted therapies against ESCC. Therefore, identifying biomarkers which may be used for early detection, prognostic stratification and novel therapeutic interventions are required to effectively treat patients with ESCC.

Melanoma-associated antigens (MAGEs) are a group of well-characterized members of the cancer/testis antigen (CTA) family, which are expressed in a number of types of malignant tumors, including hepatocellular carcinoma, breast cancer, renal carcinoma and non-small cell lung cancer (5-8). MAGE-A is a subfamily of MAGEs and MAGE-A is highly homologous with the MAGE gene on chromosome $\mathrm{X}$ in CTAs (9). In addition, MAGE-A genes have been identified to be expressed at increased levels in a number of types of malignant tumor, but are rarely expressed in healthy tissues (10). The biological functions of MAGE genes remain unknown, therefore MAGE-A may be identified as a target for cancer immunotherapy. MAGE-A9 has been studied in a number of types of cancer, including hepatocellular carcinoma, breast cancer, renal carcinoma, non-small cell lung cancer and 


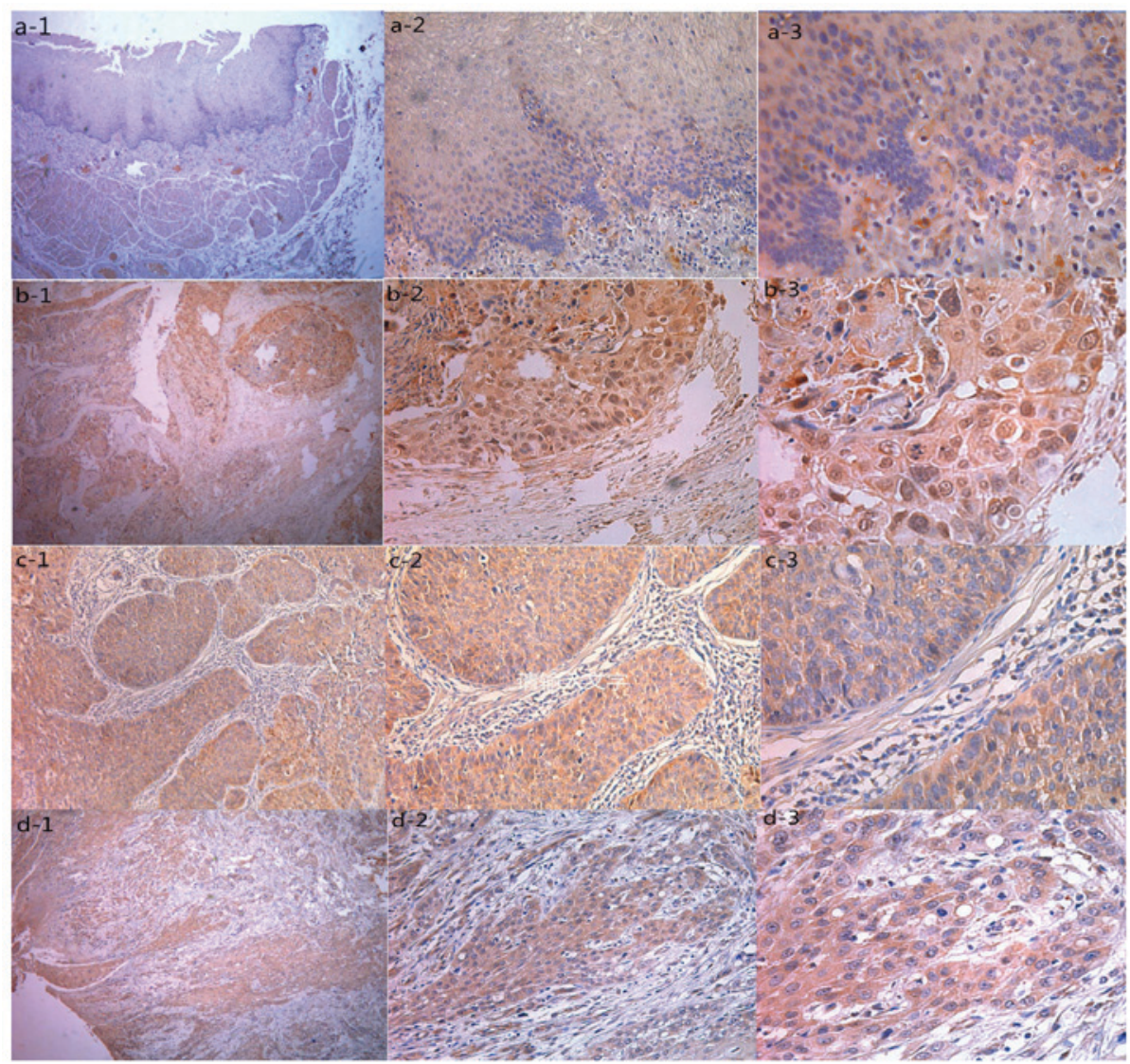

Figure 1. Representative pattern of MAGE-A9 protein expression in ESCC and normal tissues in a tissue microarray. (A) Negative IHC staining of MAGE-A9 of non-cancerous tissue (magnifications: 1, x40; 2, x200; 3, x400). (B) IHC staining of MAGE-A9 of well-differentiated ESCC tissue (magnifications: 1, x40; 2, x200; 3, x400). (C) High IHC staining of MAGE-A9 of moderately-poorly differentiated ESCC tissue (magnifications: 1, x40; 2, x200; 3, x400). (D) IHC staining of MAGE-A9 of well-differentiated ESCC sample (magnifications: 1, x40; 2, x200; 3, x400). MAGE-A9, melanoma-associated antigen-9; ESCC, esophageal squamous cell carcinoma; IHC, immunohistochemistry.

laryngeal squamous cell carcinoma (11-14). MAGE-A9 has been associated with tumor recurrence and progression in bladder cancer (15). The results of the present study revealed that MAGE-A9 was involved in the progress of malignant tumors, but the association between MAGE-A9 expression and the clinicopathological outcome in ESCC remains unknown.

In the present study, the expression level of MAGE-A9 protein and mRNA in ESCC and normal tissues was determined using immunohistochemistry (IHC) and the quantitative polymerase chain reaction (qPCR). Furthermore, the association between MAGE-A9 expression and the clinicopathological features observed in patients with ESCC, and the prognostic significance, were analyzed.

\section{Materials and methods}

Patient specimens. A total of 103 paraffin-embedded ESCC tissues and 30 healthy tissue samples were collected from the archives of the Department of Pathology at The First Affiliated Hospital of Zhengzhou University (Zhengzhou, China), between August 2011 and January 2012. All patients were staged in accordance with the tumor-node-metastasis classification system (Union for International Cancer Control 2009) (16). Prior to surgery, none of the patients had received neoadjuvant chemotherapy, radiation therapy or immunotherapy. Clinical data, including sex, age, smoking history, alcohol, tumor size, pathological grade, lymph node metastasis and the degree of infiltration, were collected. The present study was approved by the Ethics Committee of The First Affiliated Hospital of Zhengzhou University (Zhengzhou, China).

Tissue microarray (TMA) construction and IHC analysis. A total of 54 ESCC tissues and 30 healthy tissues were collected, and TMAs were produced by the Department of Clinical Pathology, The First Affiliated Hospital of Zhengzhou University (Zhengzhou, China). The TMA was sectioned (thickness, $4 \mathrm{um}$ ) and incubated at $60^{\circ} \mathrm{C}$ for $3 \mathrm{~h}$. Slides were subsequently deparaffinized with xylene, rehydrated in an ethanol series, submerged in $0.01 \mathrm{M}$ citrate buffer $(\mathrm{pH}, 6.0)$ antigen retrieval buffer and microwaved for antigenic retrieval. Tissues were blocked with $3 \% \mathrm{H}_{2} \mathrm{O}_{2}$ for $20 \mathrm{~min}$ at room temperature, followed by incubation with goat serum for $20 \mathrm{~min}$ at room temperature. Subsequently, samples were incubated at $4^{\circ} \mathrm{C}$ with human anti-MAGEA9 immunoglobulin $\mathrm{G}$ antibody (catalog no. ab38493; dilution, 1:50; Abcam, Cambridge, UK) in a dark box overnight, followed by incubation at $37^{\circ} \mathrm{C}$ with a biotinylated anti-rabbit secondary antibody (catalog no. ab150077; dilution, 1:50; Abcam) for $20 \mathrm{~min}$. As a negative control, samples were incubated with PBS instead of a specific antibody. The sections were incubated with 
Table I. Association between increased MAGE-A9 expression and the clinicopathological characteristics in esophageal squamous cell carcinoma.

\begin{tabular}{|c|c|c|c|c|c|}
\hline \multirow[b]{2}{*}{ Group } & \multicolumn{3}{|c|}{ MAGE-A9 } & \multirow[b]{2}{*}{$\chi^{2}$} & \multirow[b]{2}{*}{ P-value } \\
\hline & $\mathrm{n}$ & + & $\%$ & & \\
\hline Total & 103 & 57 & 55.3 & & \\
\hline \multicolumn{6}{|l|}{ Sex } \\
\hline Male & 75 & 45 & 60.0 & 2.424 & 0.119 \\
\hline Female & 28 & 12 & 42.9 & & \\
\hline \multicolumn{6}{|c|}{ Age, years } \\
\hline$\leq 60$ & 42 & 24 & 57.1 & 0.093 & 0.917 \\
\hline$>60$ & 61 & 33 & 54.1 & & \\
\hline \multicolumn{6}{|l|}{ Smoking } \\
\hline No & 51 & 25 & 49.0 & 1.633 & 0.201 \\
\hline Yes & 52 & 32 & 61.5 & & \\
\hline \multicolumn{6}{|l|}{ Alcohol } \\
\hline No & 81 & 44 & 54.3 & 0.159 & 0.690 \\
\hline Yes & 22 & 13 & 59.1 & & \\
\hline \multirow{2}{*}{\multicolumn{6}{|c|}{$\begin{array}{l}\text { Tumor size, } \\
\mathrm{cm}\end{array}$}} \\
\hline & & & & & \\
\hline$\leq 3$ & 48 & 21 & 43.8 & 4.886 & $0.027^{\mathrm{a}}$ \\
\hline$>3$ & 55 & 36 & 65.5 & & \\
\hline \multicolumn{6}{|l|}{ pT } \\
\hline $\mathrm{T} 1$ & 12 & 4 & 33.3 & 2.717 & 0.257 \\
\hline $\mathrm{T} 2$ & 37 & 21 & 56.8 & & \\
\hline $\mathrm{T} 3$ & 54 & 32 & 59.3 & & \\
\hline \multicolumn{6}{|c|}{$\begin{array}{l}\text { Lymph node } \\
\text { metastasis }\end{array}$} \\
\hline No & 69 & 32 & 46.4 & 6.794 & $0.009^{\mathrm{a}}$ \\
\hline Yes & 34 & 25 & 73.5 & & \\
\hline \multicolumn{6}{|c|}{$\begin{array}{l}\text { Pathological } \\
\text { grade }\end{array}$} \\
\hline I & 10 & 4 & 40.0 & 9.606 & $0.008^{\mathrm{a}}$ \\
\hline II & 70 & 23 & 32.9 & & \\
\hline III & 23 & 16 & 69.9 & & \\
\hline
\end{tabular}

${ }^{\mathrm{a}} \mathrm{P}<0.05$. MAGE-A9, melanoma-associated antigen-9; $\mathrm{pT}$, pathological tumor stage.

biotinylated horseradish peroxidase-labeled streptavidin. The reaction was revealed using 3,3-diaminobenzidine, and finally the sections were stained with hematoxylin and incubated at room temperature for $4 \mathrm{~min}$. Results were determined using a light microscope (magnification, x40, x200 and x400). The proportion of MAGE-A9-positive cells was scored as follows: $0,0 \% ; 1,<30 \% ; 2$, between 30 and $70 \%$; and $3,>70 \%$. In addition, the intensity of MAGE-A9 staining was scored as follows: 0, negative staining; 1, yellow staining; 2, light brown staining; and 3, brown staining. The two scores were multiplied and used as the final staining score. The degree of cytoplasmic MAGE-A9 staining was quantified using a two-level grading system and staining scores were defined as follows: 0-1, no expression; 2-4, low expression; and 5-9, high expression.

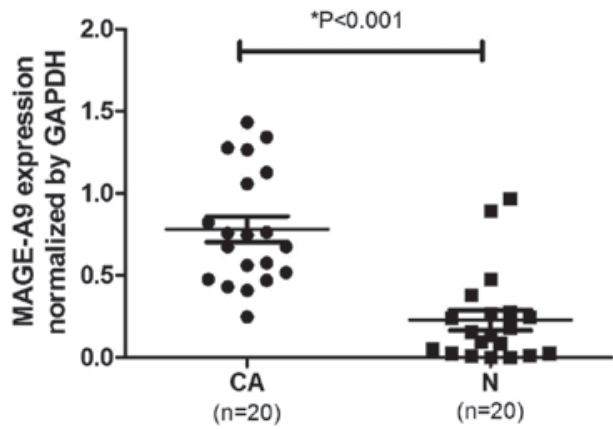

Figure 2. MAGE-A9 expression in ESCC tissues and normal tissues. The quantitative polymerase chain reaction was used to evaluate MAGE-A9 mRNA expression levels in ESCC, compared with corresponding normal tissues. When normalized to GAPDH mRNA levels, the MAGE-A9 mRNA level in ESCC tissues was significantly increased compared with that in normal tissues $(\mathrm{P}<0.05)$. MAGE-A9, melanoma-associated antigen-9; ESCC, esophageal squamous cell carcinoma; CA, ESCC tissue; N, normal tissue.

Reverse transcription (RT)-qPCR analysis. A total of 20 ESCC tissue samples and 20 corresponding healthy tissues were collected. Total RNA was extracted from tissues using TRIzol reagent (Thermo Fisher Scientific, Inc., Waltham, MA, USA), according to the manufacturer's protocol, for performing RT-PCR and qPCR analysis. Total RNA (4 $\mu \mathrm{g})$ was reverse-transcribed into cDNA using RevertAid First Strand cDNA Synthesis (Thermo Fisher Scientific, Inc.), according to the manufacturer's protocol. qPCR was performed using the Quantitative SYBR Green PCR kit (Thermo Fisher Scientific, Inc.) on an ABI PRISM 7500 Fast system (Applied Biosystems; Thermo Fisher Scientific, Inc.). The primers used were as follows: MAGE-A9 forward primer, 5'-CAC TGT ATG TCA TCT CTG-3'; reverse primer, 5'-ACT ACT GTCATT CAT TAA CT-3'; GADPH, used as a reference gene, forward primer, 5'-GTC ATC ATG TCT CTC GAG-3'; reverse primer, 5'-AAC TCA GCC ACC TTC AA-3'. qPCR amplification was conducted using the SYBR Green Master Mix (Takara Bio, Inc., Otsu, Japan). The reaction mixtures were analyzed in an ABI PRISM 7500 Fast Real-Time PCR system. The thermocycling conditions were as follows: $95^{\circ} \mathrm{C}$ for $30 \mathrm{sec}$ followed by 40 cycles of $95^{\circ} \mathrm{C}$ for $5 \mathrm{sec}$ and $60^{\circ} \mathrm{C}$ for $30 \mathrm{sec}$. All samples were analyzed for 3 parallel samples in triplicate. The relative expression of the target gene was calculated using $2^{-\Delta \Delta C q}$ values (17).

Statistical analysis. All statistical analyses were conducted using PrismDemo (version 5.0; GraphPad Software, Inc., La Jolla, CA, USA) and SPSS (version 17.0; SPSS, Inc., Chicago, IL, USA). The MAGE-A9 mRNA expression in ESCC tissues, compared with matched healthy tissues, was analyzed using the Wilcoxon signed rank non-parametric test. The significance of MAGE-A9 protein expression on clinicopathological parameters of ESCC was determined using the $\chi^{2}$ test. The Kaplan-Meier estimator survival method was used to evaluate the associations between MAGE-A9 expression and the outcome for patients with ESCC. Cox's proportional hazards regression models was used to determine factors that were independently associated with overall survival rates. For all tests, $\mathrm{P}<0.05$ was considered to indicate a statistically significant difference. 
Table II. Univariate and multivariate analysis of the association of prognostic factors in esophageal squamous cell carcinoma with overall survival.

\begin{tabular}{|c|c|c|c|c|c|c|}
\hline \multirow[b]{2}{*}{ Characteristic } & \multicolumn{3}{|c|}{ Univariate analysis } & \multicolumn{3}{|c|}{ Multivariate analysis } \\
\hline & $\mathrm{RR}$ & P-value & $95 \% \mathrm{Cl}$ & RR & P-value & $95 \% \mathrm{Cl}$ \\
\hline \multicolumn{7}{|l|}{ Sex } \\
\hline Male vs. female & 1.794 & 0.138 & $0.828-3.887$ & & & \\
\hline $\begin{array}{l}\text { Age, years } \\
\leq 60 \text { vs. }>60\end{array}$ & 0.821 & 0.531 & $0.443-1.522$ & & & \\
\hline \multicolumn{7}{|l|}{ Smoking } \\
\hline No vs. yes & 1.237 & 0.497 & $0.670-2.287$ & & & \\
\hline \multicolumn{7}{|l|}{ Alcohol } \\
\hline $\begin{array}{l}\text { No vs. yes } \\
\text { Tumor size, cm }\end{array}$ & 1.440 & 0.290 & $0.124-2.001$ & & & \\
\hline $\begin{array}{l}\leq 3 \text { vs. }>3 \\
\text { pT }\end{array}$ & 2.058 & $0.041^{\mathrm{a}}$ & $1.029-4.114$ & 1.322 & 0.449 & $0.642-2.721$ \\
\hline $\mathrm{T} 1$ and $\mathrm{T} 2$ vs. $\mathrm{T} 3$ & 1.493 & 0.102 & $0.924-2.414$ & & & \\
\hline $\begin{array}{l}\text { Pathological grade } \\
\text { II and II vs. III }\end{array}$ & 1.344 & 0.267 & $0.797-2.264$ & & & \\
\hline Lymph node metastas & & & & & & \\
\hline $\begin{array}{l}\text { No vs. yes } \\
\text { MAGE-A9 }\end{array}$ & 5.76 & $0.001^{\mathrm{a}}$ & $3.043-10.905$ & 4.256 & $0.001^{\mathrm{a}}$ & $2.161-8.380$ \\
\hline High vs. low & 4.067 & $0.001^{\mathrm{a}}$ & $1.932-8.560$ & 2.93 & $0.006^{\mathrm{a}}$ & $1.367-6.277$ \\
\hline
\end{tabular}

${ }^{\mathrm{a}} \mathrm{P}<0.05$. MAGE-A9, melanoma-associated antigen-9; pT, pathological tumor stage; RR, relative risk; CI, confidence interval.
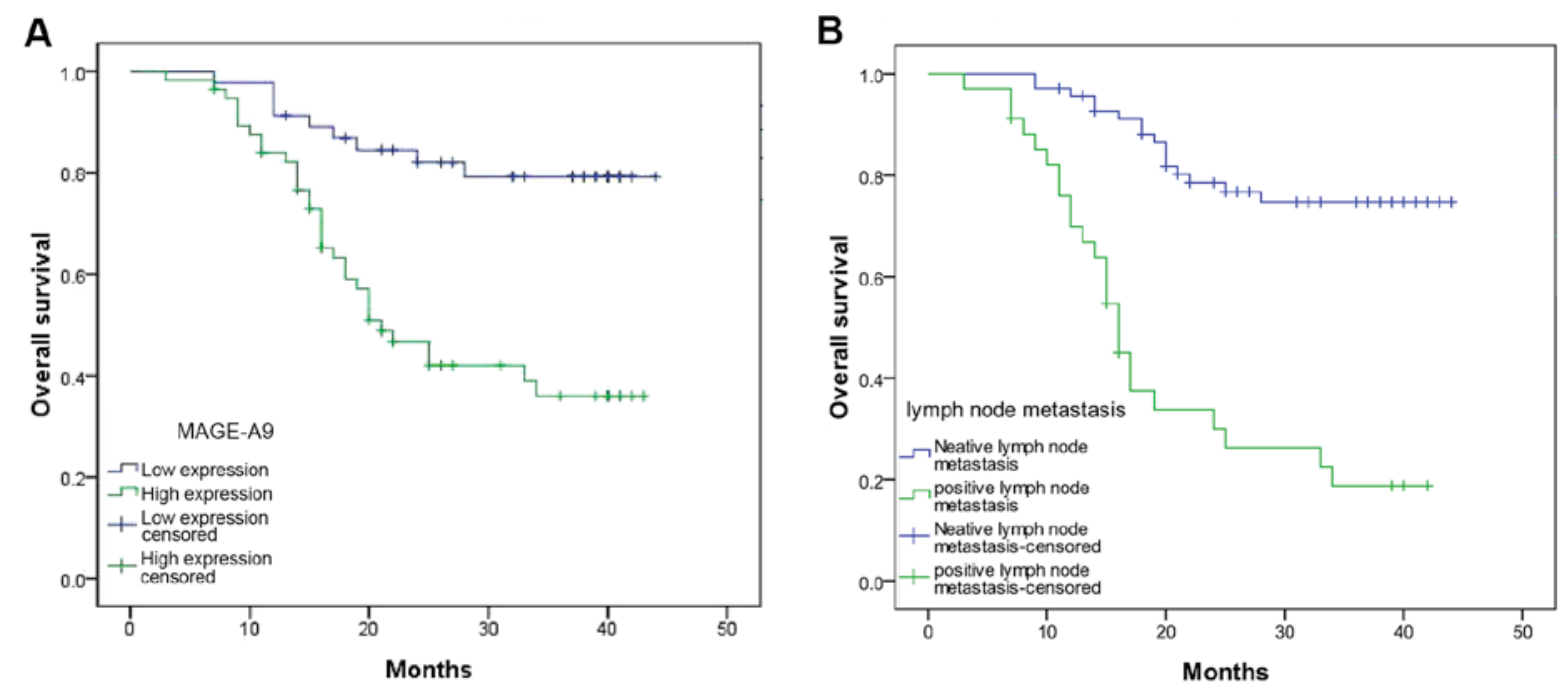

Figure 3. Survival analysis of patients with esophageal squamous cell carcinoma using the Kaplan-Meier estimator method. (A) The overall survival rate in patients with increased MAGE-A9 expression (green line) was significantly decreased, compared with that in patients with decreased or no MAGE-A9 expression (blue line). (B) The overall survival rate in patients with lymph node metastasis (green line) was significantly decreased compared with that in patients without lymph node metastasis (blue line). MAGE-A9, melanoma-associated antigen-9.

\section{Results}

Determination of MAGE-A9 protein expression in ESCC using IHC. IHC was used to determine the expression levels of MAGE-A9. Increased MAGE-A9 expression was determined in 57/103 (55.34\%) ESCC tissues and 4/30 (13.3\%) non-cancerous healthy tissues. A significant difference was determined in the increased expression levels of MAGE-A9 protein between ESCC tissues and healthy tissues $(\mathrm{P}<0.05)$. MAGE-A9 positive staining was predominantly localized in the cytoplasm of cancer cells. The typical ESCC staining for MAGE-A9 expression in ESCC is presented in Fig. 1. The 
association between MAGE-A9 expression and the clinicopathological characteristics of 103 patients with ESCC is presented in Table I. Increased MAGE-A9 expression levels were associated with the pathological grade $(\mathrm{P}=0.008)$, tumor size $(\mathrm{P}=0.027)$ and lymph node metastasis $(\mathrm{P}=0.009)$. In comparison, no significant associated was discovered between MAGE-A9 expression and other clinical characteristics, including sex, age, smoking status, alcohol consumption, Tumor-Node-Metastasis stage (16) and histopathological grade (Table I).

Analysis of MAGE-A9 $m R N A$ expression in ESCC using $q P C R$. Total RNA was extracted from the ESCC tissues and corresponding non-cancerous tissues, and qPCR was used to evaluate MAGE-A9 mRNA expression. As presented in Fig. 2, the mean \pm standard error of MAGE-A9 mRNA in ESCC tissues $(0.722 \pm 0.075)$ was significantly increased compared with that of the corresponding non-cancerous tissues $(0.223 \pm 0.067)$, when normalized to GAPDH (P<0.05; Fig. 2).

Survival analysis. Univariate analysis demonstrated that the overall survival of 103 patients with ESCC was associated with lymph node metastasis $(\mathrm{P}=0.001)$, tumor size $(\mathrm{P}=0.041)$ and MAGE-A9 expression $(\mathrm{P}=0.001)$ (Table II). In addition, multivariate analysis using Cox's regression model suggested that lymph node metastasis $(\mathrm{P}=0.001)$ and MAGE-A9 expression $(\mathrm{P}=0.006)$ may be independent prognostic factors for the overall survival rate. The Kaplan-Meier estimator survival curves demonstrated that patients with ESCC exhibiting lymph node metastasis and increased MAGE-A9 presented a markedly poorer survival rate (Fig. 3).

\section{Discussion}

MAGE proteins have been identified to exhibit a prognostic value in a number of tumor tissues (18-22); however, the normal physiological role of MAGE-A remains unknown and their contribution to the development of cancer is poorly understood. There is evidence that MAGE proteins are involved in the regulation of apoptosis; for example, MAGE-A3 has been identified to inhibit the activation of caspase 12 in vitro and caspase 12 in turn is capable of inducing apoptosis $(23,24)$. In addition, MAGE-A protein has been identified to inhibit the function of p53 through direct and indirect mechanisms (25). These previous studies have indicated that MAGE-As may serve an important role in human cancers, but the association between MAGE-A9 and ESCC, and whether MAGE-A9 may be used as a targeted for diagnosis and therapy against ESCC, remains unknown.

In the present study, MAGE-A9 mRNA expression in ESCC tissues was determined using RT-qPCR. The results revealed an increased expression of MAGE-A9 in ESCC tissues, compared with that in healthy tissues. In addition, IHC analysis was conducted to evaluate MAGE-A9 protein expression in ESCC TMA specimens. This analysis identified increased MAGE-A9 expression in the cytoplasm and mesenchyme of ESCC tissues, compared with that in healthy tissues. Specific parameters, including the pathological grade, lymph node metastasis and tumor size, were associated with MAGE-A9 protein expression. Univariate analysis identified that MAGE-A9 protein expression, lymph node metastasis and tumor size were associated with the overall survival rate of patients with ESCC. Multivariate analysis using Cox's regression model validated that MAGE-A9 protein expression and lymph node metastasis may serve as independent prognostic factors for overall survival. Increased MAGE-A9 expression was associated with a poor prognosis in patients with ESCC. The results of the present study were consistent with previous studies carried out in hepatocellular carcinoma and non-small cell lung cancer (7,11). Additionally, the Kaplan-Meier estimator analysis demonstrated that patients with ESCC with increased MAGE-A9 expression exhibited a markedly unfavorable outcome.

In the present study, expression levels of the protein and gene of MAGE-A9 were investigated. For MAGE-A9-positive tumors, decreased MAGE-A9 expression cannot be excluded, since gene activity may be influenced by other factors. Notably, it was identified that p53 interacts with MAGE-A9 and the underlying molecular mechanism which may be investigated using gene knockdown or gene knockout of MAGE-A9. Additional analysis of the mechanism of MAGE-A9 expression in ESCC cells is required.

The present study is the first, to the best of our knowledge, to demonstrate an increased expression of MAGE-A9 in ESCC tissues, which exhibited an association with the poor survival of patients with ESCC. MAGE-A9 may provide a therapeutic strategy for ESCC treatment.

\section{References}

1. Siegel RL, Miller KD and Jemal A: Cancer statistics, 2015. CA Cancer J Clin 65: 5-29, 2015.

2. He J and Chen WQ: Chinese cancer registry annual report. Military Medical Science Press, Beijing, 2012: P56-58, 2012 (In Chinese).

3. Bosset JF, Gignoux M, Triboulet JP, Tiret E, Mantion G, Elias D, Lozach P, Ollier JC, Pavy JJ, Mercier M and Sahmoud T: Chemoradiotherapy followed by surgery compared with surgery alone in squamous-cell cancer of the esophagus. N Engl J Med 337: 161-167, 1997.

4. Zhang Y: Epidemiology of esophageal cancer. World J Gastroenterol 19: 5598-6006, 2013.

5. Roch N, Kutup A, Vashist Y, Yekebas E, Kalinin V and Izbicki JR: Coexpression of MAGE-A peptides and HLA class I molecules in hepatocellular carcinoma. Anticancer Res 30: 1617-1623, 2010.

6. Lian Y, Sang M, Ding C, Zhou X, Fan X, Xu Y, Lü W and Shan B: Expressions of MAGE-A10 and MAGE-A11 in breast cancers and their prognostic significance: A retrospective clinical study. J Cancer Res Clin Oncol 138: 519-527, 2012.

7. Hatiboglu G, Pritsch M, Macher-Goeppinger S, Zöller M, Huber J, Haferkamp A, Pahernik S, Wagener N and Hohenfellner M: Prognostic value of melanoma-associated antigen A9 in renal cell carcinoma. Scand J Urol 47: 311-322, 2012.

8. Melloni G, Ferreri AJ, Russo V, Gattinoni L, Arrigoni G, Ceresoli GL, Zannini P and Traversari C: Prognostic significance of cancer-testis gene expression in resected non-small cell lung cancer patients. Oncol Rep 12: 145-151, 2004.

9. Chen YT, Chiu R, Lee P, Beneck D, Jin B and Old LJ: Chromosome $\mathrm{X}$-encoded cancer/testis antigens show distinctive expression patterns in developing gonads and in testicular seminoma. Hum Reprod 26: 3232-3243, 2011.

10. Meek DW and Marcar L: MAGE-A antigens as targets in tumour therapy. Cancer Lett 324: 126-132, 2012.

11. Gu X, Fu M, Ge Z, Zhan F, Ding Y, Ni H, Zhang W, Zhu Y, Tang X, Xiong L, et al: High expression of MAGE-A9 correlates with unfavorable survival in hepatocellular carcinoma. Sci Rep 4: 6625: 2014

12. Xu X, Tang X, Lu M, Tang Q, Zhang H, Zhu H, Xu N, Zhang D, Xiong L, Mao Y and Zhu J: Overexpression of MAGE-A9 predicts unfavorable outcome in breast cancer. Exp Mol Pathol 97: 579-584, 2014. 
13. Zhang S, Zhai X, Wang G, Feng J, Zhu H, Xu L, Mao G and Huang J: High expression of MAGE-A9 in tumor and stromal cells of non-small cell lung cancer was correlated with patient poor survival. Int J Clin Exp Pathol 8: 541-550, 2015.

14. Han L, Jiang B, Wu H, Zhang S and Lu X: Expression and prognostic value of MAGE-A9 in laryngeal squamous cell carcinoma. Int J Clin Exp Pathol 7: 6734-6742, 2014.

15. Bergeron A, Picard V,LaRue H,HarelF, Hovington H,Lacombe L and Fradet Y: High frequency of MAGE-A4 and MAGE-A9 expression in high-risk bladder cancer. Int J Cancer 125: 1365$1371,2009$.

16. Sobin L, Gospodarowicz M and Wittekind C (eds): TNM classification of malignant tumors. 7th edition. New Jersey, Wiley-Blackwell, 2009.

17. Schmittgen TD and Livak KJ: Analyzing real-time PCR data by the comparative C(T) method. Nat Protoc 3: 1101-1108, 2008.

18. Jungbluth AA, Busam KJ, Kolb D, Iversen K, Coplan K, Chen YT, Spagnoli GC and Old LJ: Expression of MAGE-antigens in normal tissues and cancer. Int J Cancer 85: 460-465, 2000.

19. Svobodová S, Browning J, MacGregor D, Pollara G, Scolyer RA Murali R, Thompson JF, Deb S, Azad A, Davis ID and Cebon JS: Cancer-testis antigen expression in primary cutaneous melanoma has independent prognostic value comparable to that of Breslow thickness, ulceration and mitotic rate. Eur J Cancer 47: 460-469, 2011.
20. Ogata K, Aihara R, Mochiki E, Ogawa A, Yanai M, Toyomasu Y, Ando H, Ohno T, Asao T and Kuwano H: Clinical significance of melanoma antigen-encoding gene-1 (MAGE-1) expression and its correlation with poor prognosis in differentiated advanced gastric cancer. Ann Surg Oncol 18: 1195-1203, 2011.

21. Jeon CH, Shin IH, Park JB and Chae HD: Prognostic significance of MAGE in peritoneal washes in gastric carcinoma patients without peritoneal metastasis: Results of a 5-year follow-up study. J Clin Gastroenterol 44: 682-686, 2010.

22. Pastorcic-Grgic M, Sarcevic B, Dosen D, Juretic A, Spagnoli GC and Grgic M: Prognostic value of MAGE-A and NY-ESO-1 expression in pharyngeal cancer. Head Neck 32: 1178-1184, 2010.

23. Sang M, Wang L, Ding C, Zhou X, Wang B, Wang L, Lian Y and Shan B: Melanoma-associated antigen genes-an update. Cancer Lett 302: 85-90, 2011.

24. Morishima N, Nakanishi K, Takenouchi H, Shibata T and Yasuhiko Y: An endoplasmic reticulum stress-specific caspase cascade in apoptosis cytochrome c-independent activation of caspase- 9 by caspase-12. J Biol Chem 277: 34287-34294, 2002.

25. Marcar L, MacLaine NJ, Hupp TR and Meek DW: Mage-A cancer/testis antigens inhibit p53 function by blocking its interaction with chromatin. Cancer Res 70: 10362-10370, 2010. 